\title{
Erratum: Mitochondrial SSBP1 protects cells from proteotoxic stresses by potentiating stress-induced HSF1 transcriptional activity
}

Ke Tan, Mitsuaki Fujimoto, Ryosuke Takii, Eiichi Takaki, Naoki Hayashida \& Akira Nakai

Nature Communications 6:6580 doi: 10.1038/ncomms7580 (2015); Published 12 Mar 2015; Updated 17 Apr 2015

In Supplementary Fig. 4a in this Article, the western blot representing the actin control for MEF cells in the right hand panel was inadvertently duplicated from the HeLa experiment in the left hand panel. The correct version of Supplementary Fig. 4 appears below.

a

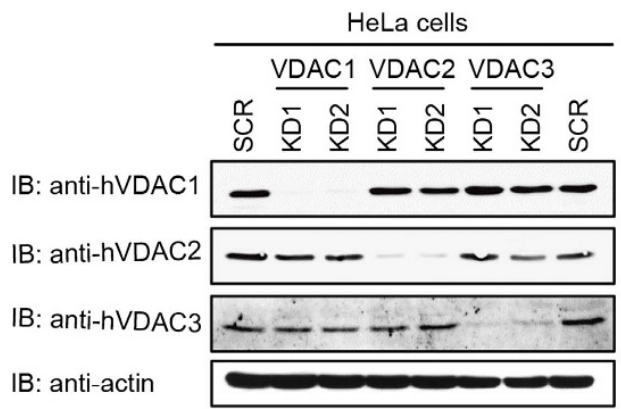

b

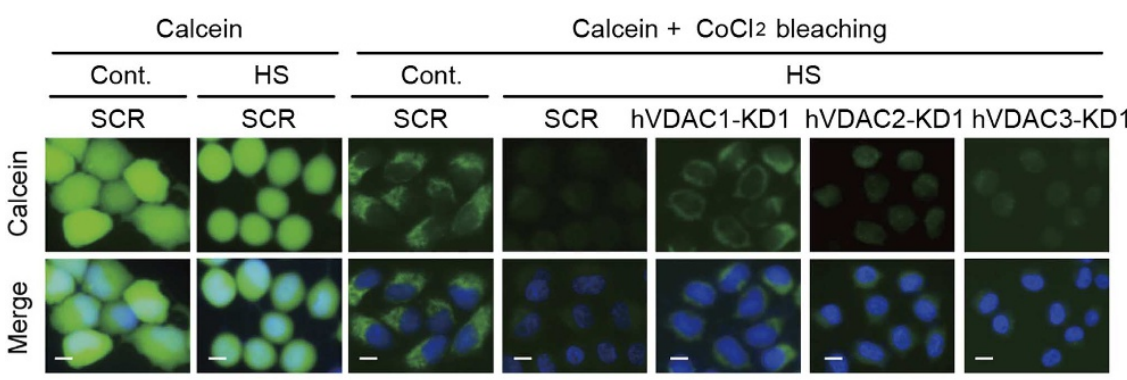

Supplementary Figure 4 |


Calcein $+\mathrm{CoCl}_{2}$ 